\title{
THE CRUCIAL ROLE OF NEUTROPHIL GRANULOCYTES IN BONE FRACTURE HEALING
}

\author{
A. Kovtun ${ }^{1, *}$, S. Bergdolt ${ }^{1}$, R. Wiegner ${ }^{2}$, P. Radermacher ${ }^{3}$, M. Huber-Lang ${ }^{2}$ and A. Ignatius ${ }^{1}$ \\ ${ }^{1}$ Institute of Orthopaedic Research and Biomechanics, Centre for Trauma Research Ulm, University of Ulm, \\ D-89081 Ulm, Germany \\ ${ }^{2}$ Department of Orthopaedic Trauma, Hand, Plastic and Reconstruction Surgery, Ulm University Hospital, \\ D-89081 Ulm, Germany \\ ${ }^{3}$ Institute of Anaesthesiological Pathophysiology and Process Engineering, Ulm University Hospital, D-89081 Ulm, \\ Germany
}

\begin{abstract}
Delayed bone fracture healing and the formation of nonunions represent an important clinical problem, particularly in polytrauma patients who suffer from posttraumatic systemic inflammation. However, the underlying pathomechanisms remain unclear. Neutrophil granulocytes are crucial effector cells in the systemic immune response and represent the most abundant immune cell population in the early fracture haematoma. Here we investigated the role of neutrophils in a mouse model of uncomplicated fracture healing and compromised fracture healing induced by an additional thoracic trauma. Twenty four hours before injury, $50 \%$ of the mice were systemically treated with an anti-Ly-6G-antibody to reduce neutrophil numbers. In the isolated fracture model, Ly-6G-Ab treatment significantly increased the concentration of both proand anti-inflammatory cytokines, including interleukin (IL)-6 and IL-10, and chemokines, for example, C-X-C motif ligand 1 (CXCL1) and monocyte chemotactic protein-1 (MCP-1), in the fracture haematoma. Monocyte/ macrophage recruitment was also significantly enhanced. After $21 \mathrm{~d}$, bone regeneration was considerably impaired as demonstrated by significantly diminished bone content and impaired mechanical properties of the fracture callus. These results indicate that undisturbed neutrophil recruitment and function in the inflammatory phase after fracture is crucial to initiate downstream responses leading to bone regeneration. In the combined trauma model, the reduction of neutrophil numbers ameliorated pulmonary inflammation but did not provoke any significant effect on bone regeneration, suggesting that neutrophils may not play a crucial pathomechanistic role in compromised fracture healing induced by an additional thoracic trauma.
\end{abstract}

Keywords: Fracture healing, severe trauma, thoracic trauma, inflammation, neutrophil granulocytes, macrophages.

*Address for correspondence:

Anna Kovtun

Institute of Orthopaedic Research and Biomechanics

University of Ulm

Helmholtzstr. 14

D-89081, Ulm, Germany

Telephone number: +49-731-500-55301

Fax number: +49-731-500-55302

E-mail: anna.kovtun@uni-ulm.de

\section{Introduction}

Delayed bone fracture healing and the formation of nonunions represent an important clinical problem. Up to $10 \%$ of all patients with fracture suffer from impaired bone healing, which often requires revision surgery associated with further health risks and additional costs (Giannoudis et al., 2007; Hak et al., 2014; Lefering et al., 2013). In particular, polytrauma patients, who can develop extensive systemic inflammation in response to severe tissue trauma, are at high risk for fracture healing complications, because the posttraumatic systemic inflammation negatively interferes with the inflammatory and regenerative processes at the fracture site (Bastian et al., 2011; Bhandari et al., 2003; Karladani et al., 2001; Pape et al., 2010).

Fracture healing is a complex process that occurs in the sequential phases of inflammation, bone formation and remodelling (Claes et al., 2012; Schmidt-Bleek et al., 2015). Initially, a fracture haematoma is formed, which is characterised by hypoxia, activation of complement and coagulation cascades, release of inflammatory cytokines, and immune cell recruitment within minutes after injury (Kolar et al., 2011; Kolar et al., 2010). The initial inflammatory phase clears the fracture site of cell debris and pathogens, thereby restricting the tissue damage. Inflammation must be properly terminated to allow the recruitment of endothelial and mesenchymal precursor cells, initiating soft callus formation and, finally, bone regeneration. Thus, a balance between pro- and antiinflammatory events is essential, because an impaired, increased, or prolonged inflammation is regarded to be detrimental for fracture healing (Schmidt-Bleek et al., 2015). In previous studies we found a strong impairment of bone healing in a rodent model of combined fracture and thoracic trauma (Kemmler et al., 2015; Recknagel et al., 2011), an experimental model of significant clinical relevance, because both injuries frequently coexist in severely injured patients (Lefering et al., 2013). In association with compromised healing, we observed considerably increased neutrophil granulocyte recruitment to the fracture haematoma, indicating that these cells may play a pathomechanistic role (Kemmler et al., 2015; Recknagel et al., 2013).

Neutrophil granulocytes migrate to injured tissues within minutes recruited by chemoattractants, including (C-X-C motif) ligand (CXCL)1-3, macrophage inflammatory protein-1 $\alpha, \mathrm{C} 5 \mathrm{a}$, and leukotriene $\mathrm{B}_{4}$ to clear danger- and pathogen-associated molecular patterns (DAMPs and PAMPs) (Furze and Rankin, 2008; Sadik et al., 2011). 
These cells phagocytose bacteria and cellular debris, form neutrophil extracellular traps (NETs), and release reactive oxygen species, antimicrobial peptides, and serine proteases as well as numerous cytokines and chemokines, including interleukin (IL)-1 $\beta$, IL-6, IL-10, monocyte chemotactic protein-1 (MCP1), and CXCL1, which modulate the inflammatory response and attract monocytes (Soehnlein et al., 2009). Under excessive inflammatory conditions, hyper-activated neutrophils exhibit altered cytokine expression and undergo massive NETosis, thereby aggravating tissue damage and impairing healing (Butterfield et al., 2006; Perl et al., 2012a; Tian et al., 2014; Wong et al., 2015). The function of neutrophil granulocytes in bone fracture healing has been poorly investigated. A few studies reported slightly improved bone regeneration when neutrophils were depleted using neutrophil-neutralising antiserum (Chung et al., 2006; Grøgaard et al., 1990). Contradicting these results, improved bone healing was also reported after inducing neutrophilia by the systemic application of granulocyte colony-stimulating factor (G-CSF) (Bozlar et al., 2005). However, the experimental approaches applied in these studies to deplete or activate neutrophils were rather unspecific.

In the present study, we selectively reduced neutrophil numbers using a specific anti-neutrophil antibody (Daley et al., 2008). Firstly, we used a highly standardised mouse model of isolated fracture to induce uneventful healing and, secondly, a combined fracture and thoracic trauma model, which results in posttraumatic systemic inflammation and compromised bone regeneration (Kemmler et al., 2015). Thereby, we addressed the following questions: 1) Do neutrophil granulocytes play a major role in fracture healing, and 2) does the reduction of neutrophils attenuate compromised fracture healing after severe trauma?

\section{Materials and Methods}

\section{Animal experiment}

All animal experiments were performed according to the international regulations for the care and use of laboratory animals and were approved by the local Ethical Committee (No. 1096, Regierungspräsidium Tübingen, Germany). Male C57BL/6J mice were purchased from Charles River (Sulzfeld, Germany) and received a standard mouse feed (ssniff ${ }^{\circledR}$ R/M-H, V1535-300, Ssniff, Soest, Germany) and water ad libitum.

When aged 12 weeks, the mice were intraperitoneally injected with $200 \mu \mathrm{L}$ of either anti-Ly-6G-antibody (LEAFTM anti-mouse Ly-6G antibody, $1 \mathrm{mg} / \mathrm{mL}$, clone 1A8, BioLegend, San Diego, CA, USA), further denoted as Ly-6G-Ab, or isotype control antibody, IgG (LEAFTM Purified Rat IgG2a, $1 \mathrm{mg} / \mathrm{mL}$, BioLegend). An osteotomy $(0.4 \mathrm{~mm})$ was performed after $24 \mathrm{~h}$ at the mid-shaft of the right femur in all the mice, which was stabilised using an external fixator (RISystem, Davos, Switzerland) under general anaesthesia with $2 \%$ isoflurane (Forene, Abbott, Wiesbaden, Germany) (Kemmler et al., 2015; Röntgen et $a l ., 2010)$. Additionally, $50 \%$ of the Ly-6G-Ab and IgGtreated mice, respectively, received an additional thoracic trauma immediately after fracture while still under general anaesthesia. In brief, a single blast wave was applied to the middle of the thorax using a blast-wave generator. This model produces a standardised bilateral, isolated lung contusion (Kemmler et al., 2015; Knöferl et al., 2003). For pain medication, all mice obtained $25 \mathrm{mg} / \mathrm{L}$ tramadol hydrochloride (Tramal $^{\circledR}$, Gruenenthal GmbH, Aachen, Germany) in the drinking water $1 \mathrm{~d}$ pre-surgery until $3 \mathrm{~d}$ post-surgery. In most cases 5-7 mice were used per group and time point, unless indicated otherwise in the figure legends.

\section{Tissue harvesting and preparation}

Animals were sacrificed at $6 \mathrm{~h}$ and 1, 3, 7 and $21 \mathrm{~d}$ after trauma under general anaesthesia through blood withdrawal via the vena cava inferior. At each time point, blood smears were Giemsa stained to count neutrophils.

\section{Plasma}

Blood was collected $6 \mathrm{~h}$ after trauma in microvettes (Microvette $^{\circledR} 100 \mu \mathrm{L}, \mathrm{K} 3$ EDTA, Sarstedt AG \& Co, Nümbrecht, Germany) and centrifuged at $4000 \times g$ for $10 \mathrm{~min}$. The plasma was collected and stored at $-80{ }^{\circ} \mathrm{C}$.

\section{Bronchoalveolar lavage (BAL) fluid}

At $6 \mathrm{~h}$ and 1 and $7 \mathrm{~d}$ after trauma, BAL fluids were collected by flushing the lungs with $500 \mu \mathrm{L}$ of ice-cold phosphatebuffered saline (PBS) as described previously (Perl et al., 2012a). BAL fluids were centrifuged at $4000 \times \mathrm{g}$ for $10 \mathrm{~min}$ and supernatants were stored at $-80{ }^{\circ} \mathrm{C}$ for further analyses, whereas pellets were analysed as Giemsa-stained smears.

\section{Lung}

The left lung lobe was removed and then either stored at $-80^{\circ} \mathrm{C}$ until homogenate preparation or immediately fixed in $4 \%$ buffered formalin and embedded in paraffin. To prepare homogenates, the lung was homogenised using a mixer mill (MM400, Retsch GmbH, Haan, Germany) and lysed in buffer (50 mM Tris- $\mathrm{HCl} \mathrm{pH} 7.5,250 \mathrm{mM} \mathrm{NaCl}$, $3 \mathrm{mM}$ ethylenediaminetetraacetic acid (EDTA), $3 \mathrm{mM}$ ethyleneglycotetraacetic acid (EGTA), $1 \%$ Triton X-100, $0.5 \% \mathrm{NP} 40,10 \%$ glycerol) containing protease inhibitors (Halt ${ }^{\mathrm{TM}}$ Protease and Phosphatase Inhibitor Single-Use Cocktail, Fisher Scientific GmbH, Schwerte, Germany) and incubated for $30 \mathrm{~min}$ on ice. After centrifugation, total protein was determined using the Pierce ${ }^{\mathrm{TM}} \mathrm{BCA}$ Protein Assay Kit (Fisher Scientific $\mathrm{GmbH}$ ) according to the manufacturer's recommendations.

\section{Fracture haematoma}

The haematoma was removed $6 \mathrm{~h}$ after trauma and stored at $-80^{\circ} \mathrm{C}$. Homogenates were prepared in lysis buffer $(10 \mathrm{mM}$ Tris pH 7.5, $10 \mathrm{mM} \mathrm{NaCl}, 0.1 \mathrm{mM}$ EDTA, $0.5 \mathrm{mM}$ Triton $\mathrm{X}-100,0.02 \% \mathrm{NaN}_{3}, 0.2 \mathrm{mM}$ phenylmethanesulphonyl fluoride) containing protease inhibitors (Halt ${ }^{\mathrm{TM}}$ Protease and Phosphatase Inhibitor Single-Use Cocktail, Fisher Scientific $\mathrm{GmbH}$ ) and further processed similarly to lung homogenates.

\section{Fractured femur}

At 1, 3 and $7 \mathrm{~d}$ after trauma, the osteotomised femora were harvested with surrounding tissues to preserve the 
haematoma and then immediately fixed in $4 \%$ buffered formalin for immuno- and histochemical staining. For biomechanical testing on day 21 , intact and fractured femora were stored in $0.9 \% \mathrm{NaCl}$ (B. Braun Melsungen AG, Melsungen, Germany) during measurement to avoid dehydration of the samples and then fixed in $4 \%$ buffered formalin for histological analysis.

\section{Cytokine analysis}

Plasma, BAL fluid, and fracture haematoma concentrations of IL-6, IL-10, MCP-1 and CXCL1 were determined using a mouse multiplex cytokine kit according to the manufacturer's protocol (Bio-Plex Pro Cytokine Assay, Bio-Rad, Hercules, CA). Data were analysed using the standard curve of cytokine standards (Bio-Plex Manager Software 4.1). All fracture haematoma cytokine values were normalised to the homogenate protein concentration. To determine physiological cytokine concentrations in plasma and BAL fluids, untreated animals were analysed as a control.

\section{Western blot analysis}

We resolved $5 \mu \mathrm{g}$ of lysate protein using SDS-PAGE and transferred it to a nitrocellulose membrane (Bio-Rad). The membranes were incubated overnight at $4{ }^{\circ} \mathrm{C}$ with anti-GAPDH (Santa Cruz Biotechnology, Dallas, USA), myeloperoxidase (MPO) and F4/80 antibodies (all from AbD Serotec, Puchheim, Germany), respectively. The membranes were incubated using horseradish peroxidaseconjugated secondary antibodies and developed in SuperSignal West Pico Chemiluminescent Substrate (Perbio Science, ThermoFisher Scientific, Waltham, USA). The protein bands were visualised using the Fusion Molecular Imaging System (Vilber Lourmat, Eberhardzell, Germany).

\section{Histomorphometry and immunohistochemistry of lungs and fracture calli}

After fixation in $4 \%$ buffered formalin for $48 \mathrm{~h}$, lungs were embedded in paraffin. Osteotomised femora were decalcified using $20 \%$ EDTA (pH 7.2-7.4) and embedded in paraffin. For histomorphometry, $6 \mu \mathrm{m}$-thick femur sections were stained using Safranin O. All femora were analysed by light microscopy (Leica DMI6000B, Leica, Heerbrugg, Switzerland) under 50-fold magnification. The osteotomy gap was defined as a region of interest (ROI). The relative amounts of osseous tissue and cartilage were evaluated by image analysis (MMAF Version 1.4.0 MetaMorph ${ }^{\circledR}$, Leica). The different immune cell populations were stained using the following antibodies and dilutions: Neutrophil granulocytes: 1:300 LEAFTM anti-mouse Ly-6G antibody, \#127620 (BioLegend) and 1:200 goat anti-rat IgG secondary antibody, \#A10517 (Life Technologies GmbH, Darmstadt, Germany); macrophages: 1:500 rat anti-mouse F4/80 antibody, \#MCA497GA (AbD Serotec) and 1:200 goat anti-rat IgG secondary antibody, \#A10517 (Life Technologies GmbH); T-cells and dendritic cells: 1:500 rabbit anti-CD8 polyclonal antibody, \#bs0648R (Bioss Inc, Woburn, MA, USA) and 1:200 goat antirabbit IgG secondary antibody, \#B2770 (Life Technologies $\mathrm{GmbH})$. Respective non-specific IgG subtypes were used as controls. For neutrophil detection, alkaline phosphatase red chromogen (Dako REAL Detection System, Dako Corp, Hamburg, Germany) was used directly as a detection substrate. To detect macrophages and T-cells, slides were first incubated with avidin-biotin complex (Vector, Burlingame, CA) for signal amplification and then detected using NovaRed (Vector Laboratories Inc, Burlingame, CA, USA) chromogen. All sections were counterstained using haematoxylin (Waldeck, Münster, Germany) and analysed under 200- or 400-fold magnification by light microscopy.

\section{Biomechanical testing of the femora}

To evaluate the mechanical competence of the healed femora, the flexural rigidity of the femora explanted on day 21 was investigated using a non-destructive threepoint bending test on a material testing machine (1454, Zwick GmbH, Ulm, Germany). Briefly, after removal of the fixator, the proximal end of the femur was fixed, whereas the femoral condyle rested unfixed on a distal bending support in the testing machine. An axial load was applied to the top of the callus at the femoral midshaft, with a maximum force of $4 \mathrm{~N}$. The flexural rigidity (EI) was calculated from the load-deflection curve as described previously (Kemmler et al., 2015; Röntgen et al., 2010).

\section{Micro-computed tomography $(\mu \mathrm{CT})$}

After biomechanical testing, fracture calli were scanned using a $\mu \mathrm{CT}$ device (Skyscan 1172, Bruker microCT, Kontich, Belgium) at $8-\mu \mathrm{m}$ resolution using $50 \mathrm{kV}$ and $200 \mathrm{~mA}$ (Kemmler et al., 2015). $\mu \mathrm{CT}$ analysis was performed using Skyscan software (NRecon, DataViewer, CTAn, all from Bruker microCT). Calibration and thresholding (642 $\mathrm{mg}$ hydroxyapatite $/ \mathrm{cm}^{3}$ as described previously (Morgan et al., 2009)) were performed in accordance with the American Society for Bone and Mineral Research (ASBMR) guidelines for $\mu \mathrm{CT}$ analysis (Parfitt et al., 1987). The osteotomy gap and whole callus between the inner pins of the external fixator were defined as volumes of interest (VOIs).

\section{Statistical analysis}

The results are presented as the mean \pm standard deviation (SD). All data were analysed in a blinded fashion. Statistic software GraphPad Prism 6 (GraphPad Software, La Jolla, CA, USA) was used to evaluate the data. Groups were tested for normal distribution using Shapiro-Wilk test, and then compared by either Kruskall-Wallis and Dunn's post-hoc test or by one-way analysis of variance (ANOVA) and Fisher's LSD post-hoc test. If several variables were analysed, we applied two-way ANOVA with either Bonferroni's or Fisher's LSD post-hoc test. Level of significance was set to $p<0.05$.

\section{Results}

\section{Efficiency of neutrophil depletion}

The number of neutrophil granulocytes was significantly reduced by $59 \pm 23 \% 1 \mathrm{~d}$ after Ly-6G-Ab treatment. The reduction of neutrophils was sustained for $8 \mathrm{~d}$. Other leukocyte populations were unaffected (data not shown). 
Table 1. Numbers of neutrophil granulocytes in the peripheral blood of mice treated with anti-mouse Ly-6G antibody (Ly-6G-Ab) or a corresponding isotype control antibody (IgG), ${ }^{*} p<0.05$ vs. pre-treatment values (day 0 ).

\begin{tabular}{|c|c|c|c|c|c|c|}
\hline \multirow{2}{*}{$\begin{array}{c}\text { Neutrophils, \%o of } \\
\text { total leukocytes }\end{array}$} & \multicolumn{7}{|c|}{ Time after treatment, days } \\
\cline { 2 - 7 } & 0 & 1 & 2 & 4 & 8 & 22 \\
\hline \multirow{2}{*}{$\mathrm{IgG}$} & $\begin{array}{c}9.6 \pm 1.0 \\
(n=11)\end{array}$ & $\begin{array}{c}10.9 \pm 4.2 \\
(n=14)\end{array}$ & $\begin{array}{c}12.4 \pm 5.0 \\
(n=7)\end{array}$ & $\begin{array}{c}9.8 \pm 4.1 \\
(n=8)\end{array}$ & $\begin{array}{c}8.4 \pm 3.0 \\
(n=9)\end{array}$ & $\begin{array}{c}8.3 \pm 1.7 \\
(n=4)\end{array}$ \\
\hline Ly-6G-Ab & $\begin{array}{c}9.7 \pm 3.7 \\
(n=11)\end{array}$ & $\begin{array}{c}4.0 \pm 2.2^{*} \\
(n=14)\end{array}$ & $\begin{array}{c}3.6 \pm 4.4^{*} \\
(n=11)\end{array}$ & $\begin{array}{c}2.9 \pm 1.1^{*} \\
(n=7)\end{array}$ & $\begin{array}{c}6.0 \pm 4.2^{*} \\
(n=11)\end{array}$ & $\begin{array}{c}8.0 \pm 3.2 \\
(n=13)\end{array}$ \\
\hline
\end{tabular}
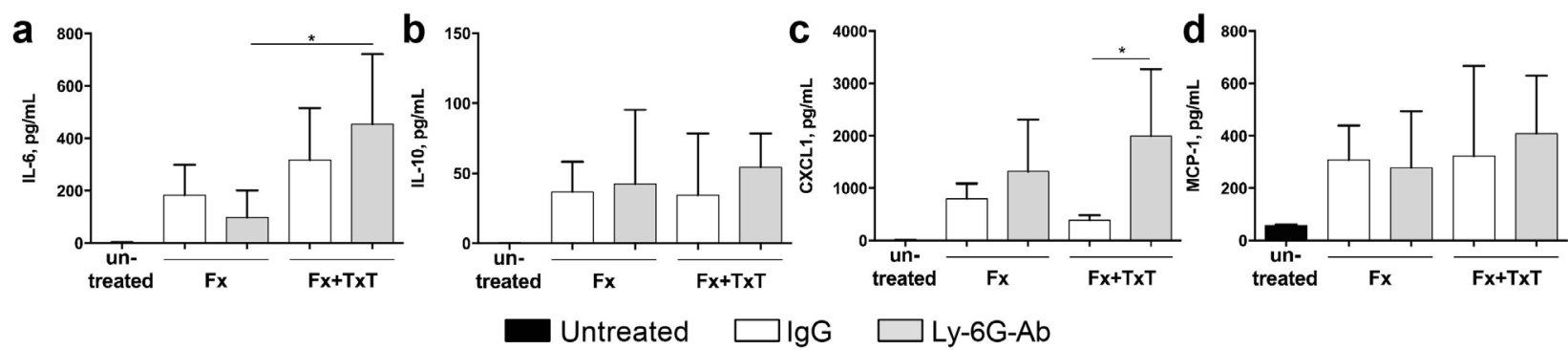

Fig. 1. Cytokine and chemokine concentrations in the plasma of control $(\mathrm{IgG})$ and Ly-6G-Ab-treated mice $6 \mathrm{~h}$ after trauma. Fx: mice with isolated fracture, Fx + TxT: mice with combined fracture and thoracic trauma; $n=5-6,{ }^{*} p<0.05$.
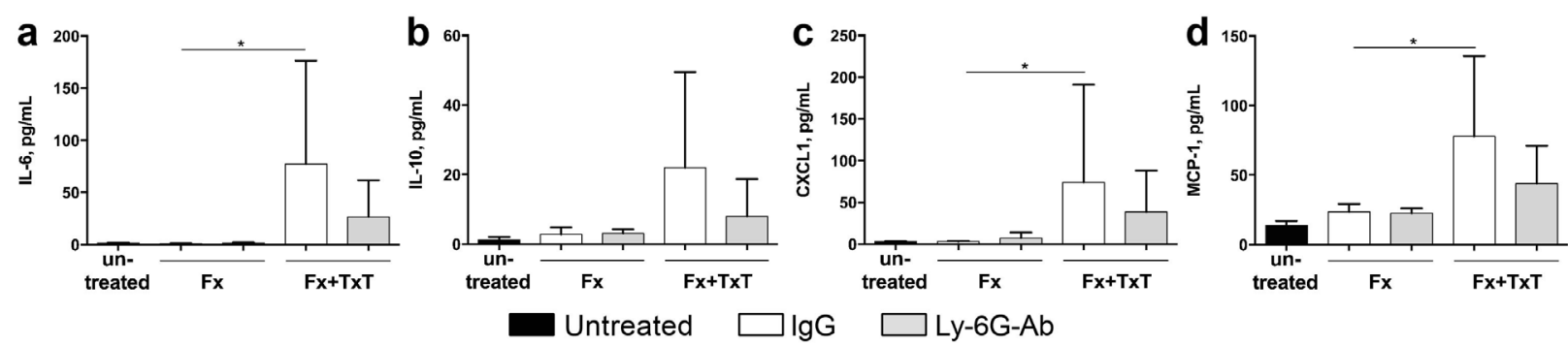

Fig. 2. Cytokine and chemokine concentrations in the BAL fluids of control (IgG) and Ly-6G-Ab-treated mice $6 \mathrm{~h}$ after trauma. Fx: mice with isolated fracture, Fx + TxT: mice with combined fracture and thoracic trauma; $n=5-6$, $* p<0.05$.

The neutrophil numbers of IgG-treated mice were not significantly altered (Table 1).

\section{Cytokine concentration in the blood plasma}

After $6 \mathrm{~h}$, plasma cytokine and chemokine levels obtained from IgG-treated mice with isolated fracture and with combined fracture and thoracic trauma were not significantly altered compared to untreated mice, indicating that there was no major systemic inflammatory response at this time point (Fig. 1). Ly-6G-Ab treatment did not significantly alter the cytokine and chemokine plasma levels in mice with fracture alone (Fig. 1). However, IL-6 was significantly increased in mice with combined trauma compared to mice with fracture alone when neutrophil numbers were reduced by Ly-6G-Ab treatment (Fig. 1). Furthermore, CXCL1 was significantly increased in Ly$6 \mathrm{G}$-Ab-treated mice compared to the IgG-treated mice after combined trauma.

\section{Inflammatory response in the lung}

We observed minimal inflammatory reaction in the lungs of mice with isolated fracture, as confirmed by unaffected BAL fluid cytokine and chemokine concentrations and low neutrophil counts (Fig. 2, 3). The additional thoracic trauma significantly increased the concentrations of IL-6, CXCL1, and MCP-1 in the BAL fluids and neutrophil infiltration into the lung tissue. Ly-6G-Ab treatment had no significant influence on lungs in the group with isolated fracture, but reduced the concentrations of inflammatory mediators and neutrophil numbers in the combined-trauma group compared to IgG-treated mice (Fig. 2, 3). Western blot analysis of the lung tissue homogenates showed a clear protein band for MPO (neutrophil marker) and F4/80 (epidermal growth factor-like module-containing mucinlike hormone receptor-like 1, monocyte/macrophage marker) in the tissue samples after combined trauma, but not after additional Ly-6G-Ab treatment (Fig. 3).

\section{Inflammatory response at the fracture site}

Analysis of inflammatory cells at the fracture site of IgGtreated control mice with isolated fracture revealed that Ly$6 \mathrm{G}^{+}$cells were abundantly present in the peripheral fracture haematoma and surrounding tissues on day 1 and that their number considerably decreased over time (Fig. 4). F4/80 cells were mainly located at the endosteal site in close proximity to the fracture gap during the early inflammatory phase (Fig. 5). At later time points, $\mathrm{F} 4 / 80^{+}$cells were also found in the peripheral callus. Low numbers of $\mathrm{CD} 8^{+}$cells 
a

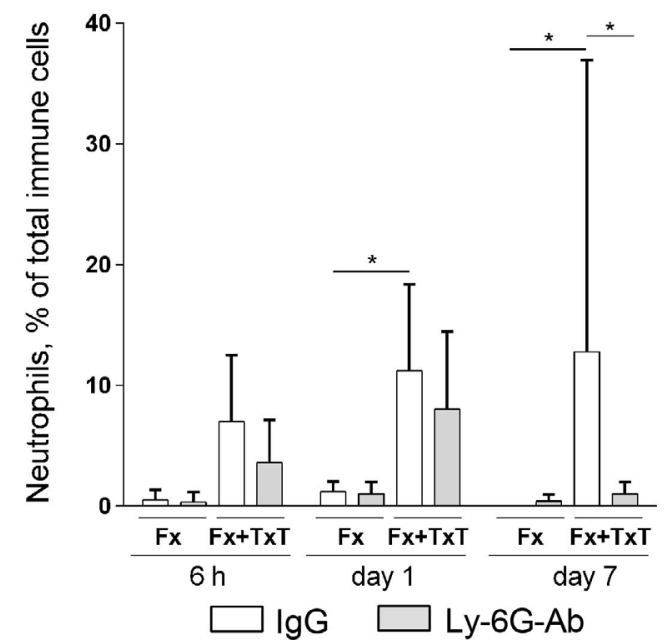

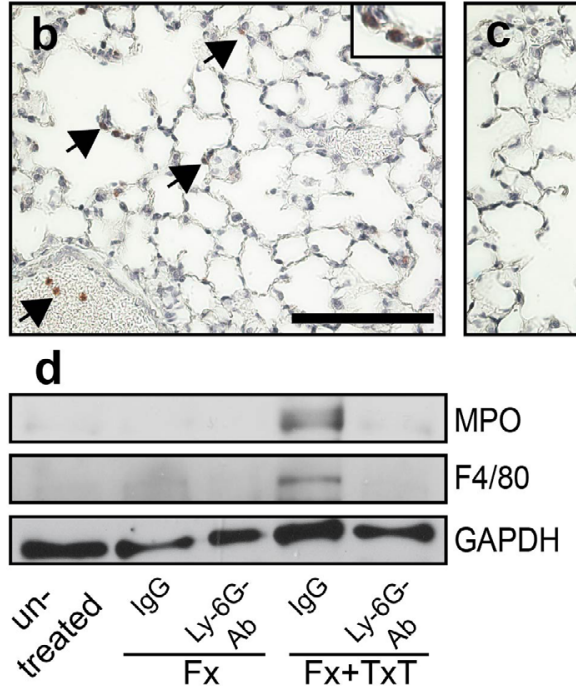

Fig. 3. Neutrophils in the BAL fluid and in lung tissue of control (IgG) and Ly-6G-Ab-treated mice. Fx: mice with isolated fracture, Fx + TxT: mice with combined fracture and thoracic trauma. (a) Number of neutrophils in the BAL fluid as determined by differential immune cell counting; $n=4-7,{ }^{*} p<0.05$. Immunohistochemical staining of neutrophils in lung tissue $7 \mathrm{~d}$ after thoracic trauma. Arrows indicate Ly-6G $\mathrm{G}^{+}$cells (b: IgG-treated group, c: Ly-6GAb-treated group). (d) Western blot analysis of lung homogenates $6 \mathrm{~h}$ after trauma (MPO: neutrophil marker, F4/80: macrophage marker, GAPDH: housekeeping protein).
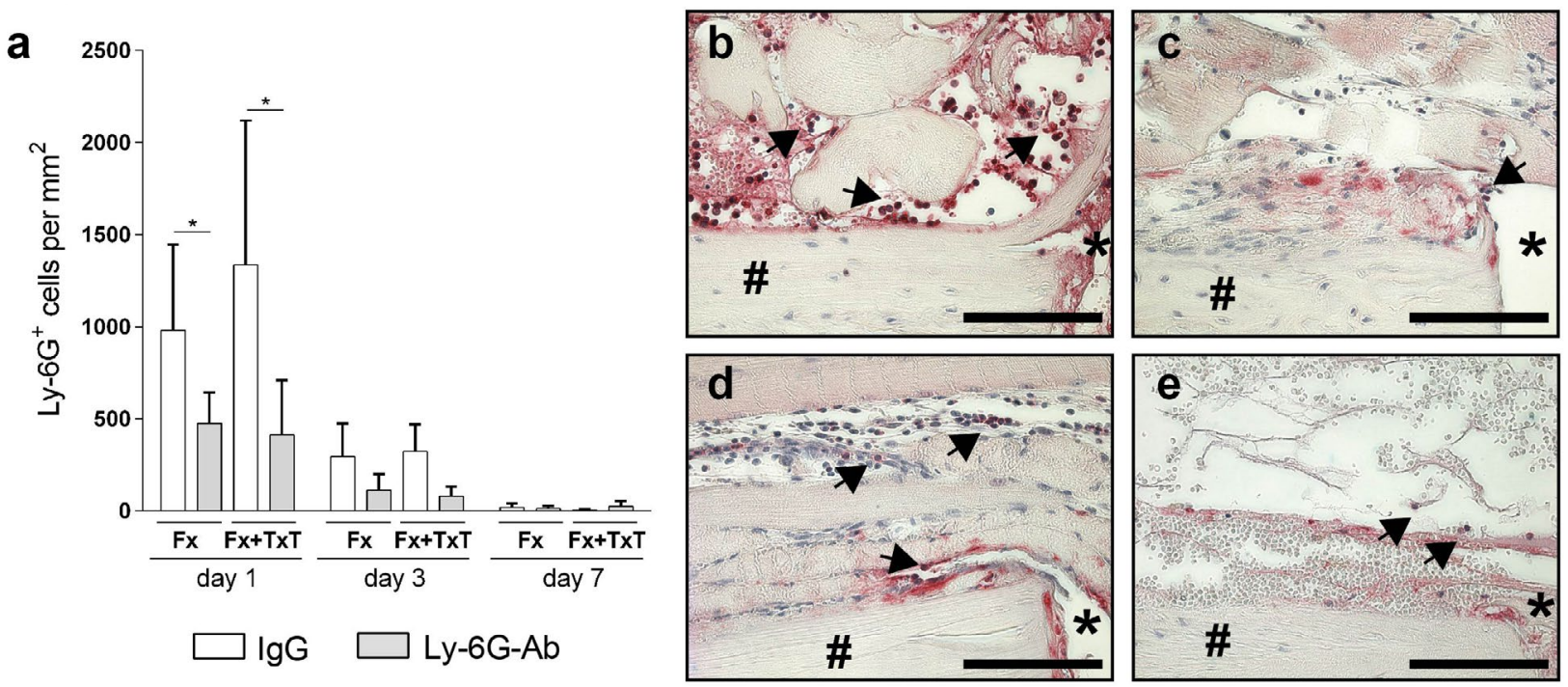

Fig. 4. Immunohistochemical staining of neutrophils in the fracture haematoma/callus of control (IgG) and Ly-6GAb-treated mice. Fx: mice with isolated fracture, Fx + TxT: mice with combined fracture and thoracic trauma. (a) Quantitative analysis of neutrophils in the fracture haematoma 1,3 and $7 \mathrm{~d}$ after trauma calculated in the periosteal area between the two inner pins of the external fixator; $n=5-6,{ }^{*} p<0.05$. Representative images of the fractured bone in the immediate proximity of the osteotomy gap $3 \mathrm{~d}$ after trauma: (b) isolated fracture; (c) isolated fracture after Ly-6G-Ab treatment; (d) combined fracture and thoracic trauma; (e) combined fracture and thoracic trauma after Ly6G-Ab treatment. Arrows indicate some of the positively stained cells, \# cortex, * fracture gap, scale bar $=100 \mu \mathrm{m}$.

were detected in the peripheral fracture haematoma on days 1 and 7 (Fig. 6). Later, their numbers increased and they were mainly present in the bone marrow-like tissue between the newly formed bone trabeculae in the peripheral callus. There their numbers were comparable with those in healthy bone marrow. The additional thoracic trauma did not significantly increase the number of $\mathrm{Ly}-6 \mathrm{G}^{+}$cells, but significantly increased the number of $\mathrm{F} 4 / 80^{+}$cells on day 3 (Fig. 4, 5). Cytokine and chemokine concentrations in the fracture haematoma were not significantly affected $6 \mathrm{~h}$ after injury (Fig. 7).

As expected, the injection of the Ly-6G-Ab significantly decreased the number of neutrophils in the fracture haematoma in the group with isolated fracture as well as in the combined-trauma group (Fig. 4). In the group with isolated fracture, neutrophil reduction significantly increased the number of $\mathrm{F} 4 / 80^{+}$cells on days 1 and 7 (Fig. 5) and elevated the levels of IL-6, IL-10, CXCL1, 

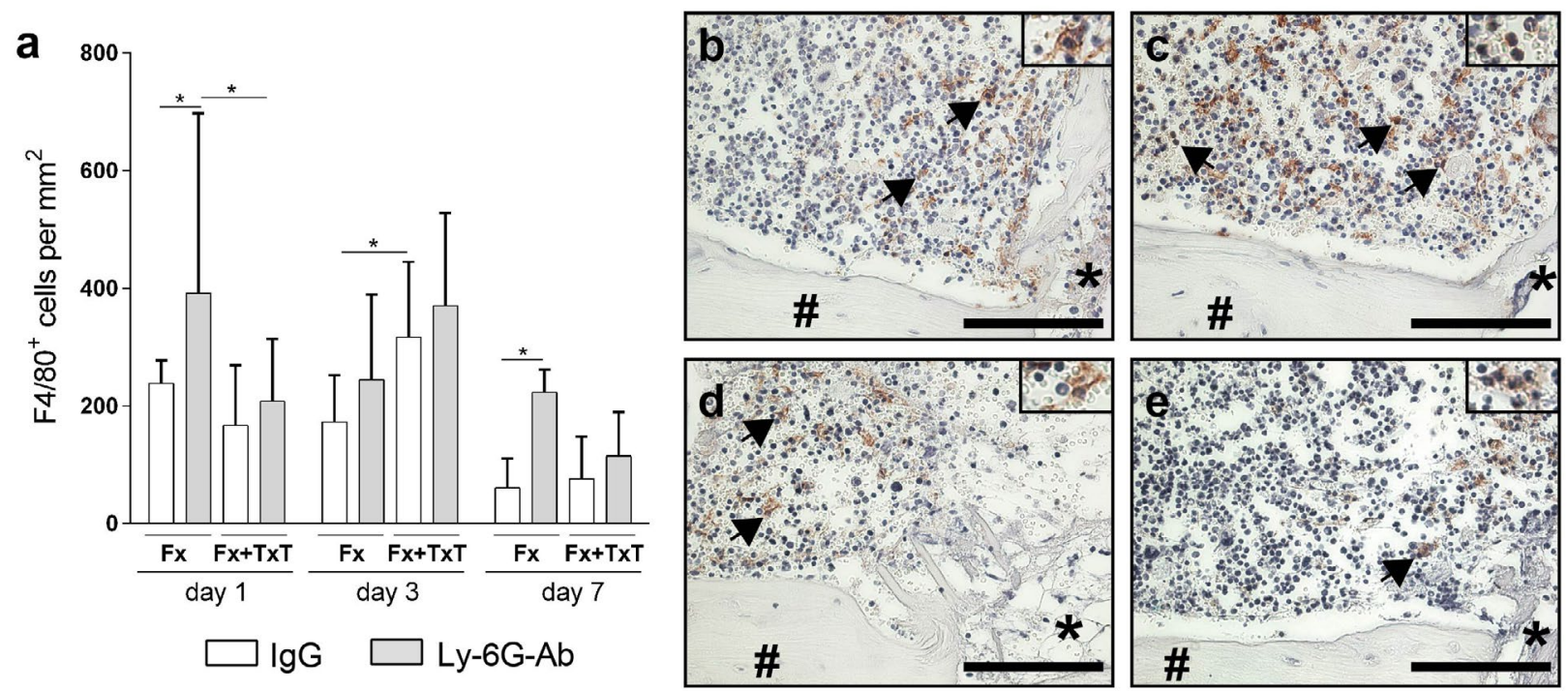

Fig. 5. Immunohistochemical staining of macrophages in the fracture haematoma/callus of control (IgG) and Ly-6GAb-treated mice using an anti-F4/80 antibody. Fx: mice with isolated fracture, Fx + TxT: mice with combined fracture and thoracic trauma. (a) Quantitative analysis of macrophages in the fracture haematoma 1, 3 and $7 \mathrm{~d}$ after trauma; $n=5-7,{ }^{*} p<0.05$. Representative images of the fractured bone in the immediate proximity of the osteotomy gap on day 1 after trauma: (b) isolated fracture; (c) isolated fracture after Ly-6G-Ab treatment; (d) combined fracture and thoracic trauma; (e) combined fracture and thoracic trauma after Ly-6G-Ab treatment. Arrows indicate some of the positively stained cells, \# cortex, * fracture gap, scale bar $=100 \mu \mathrm{m}$.
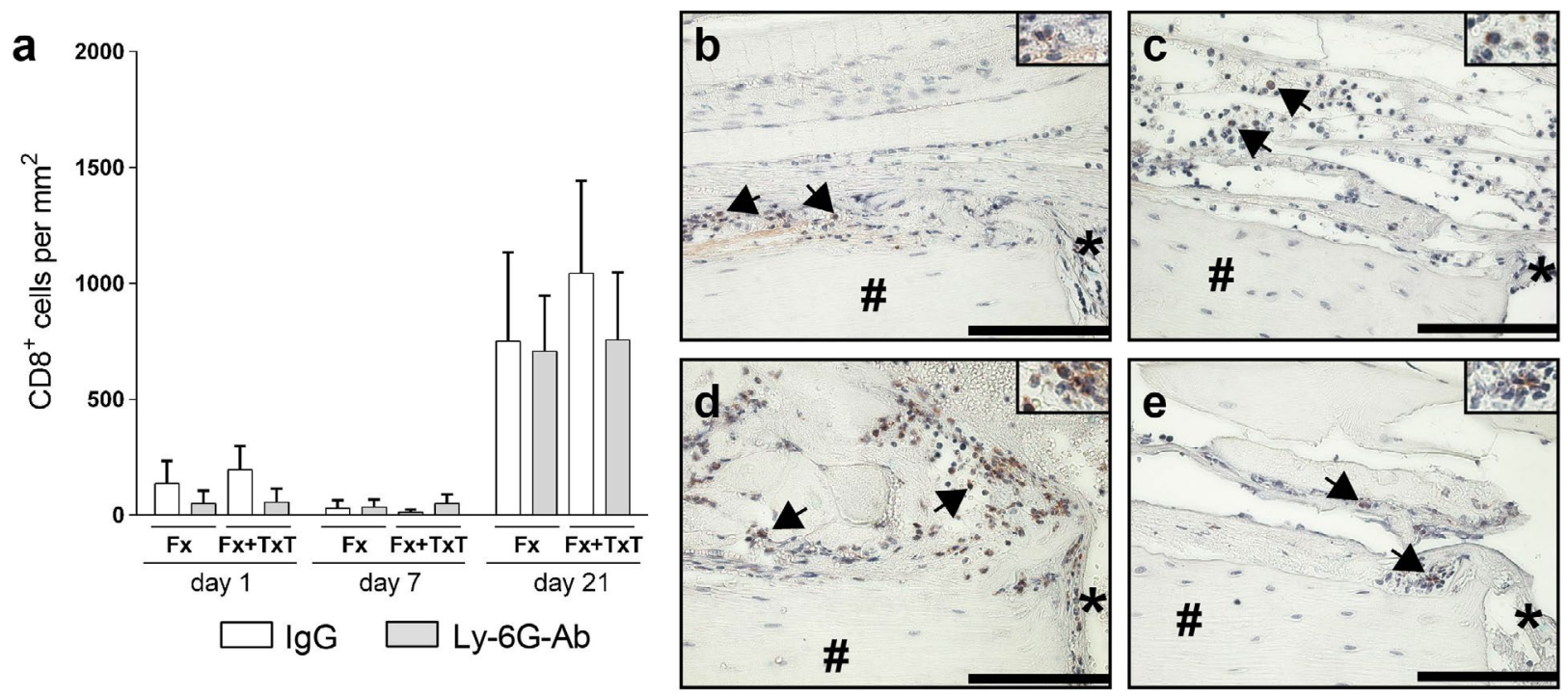

Fig. 6. Immunohistochemical staining of lymphocytes in the fracture haematoma/callus of control (IgG) and Ly-6GAb-treated mice using an anti-CD8 antibody. Fx: mice with isolated fracture, Fx + TxT: mice with combined fracture and thoracic trauma. (a) Quantitative analysis of $\mathrm{CD}^{+}$cells 1, 7 and $21 \mathrm{~d}$ after trauma calculated in the periosteal area between the two inner pins of the external fixator; $n=5-6$. Representative images of the fractured bone in the immediate proximity of the osteotomy gap on day 1 after trauma: (b) isolated fracture; (c) isolated fracture after Ly-6G-Ab treatment; (d) combined fracture and thoracic trauma; (e) combined fracture and thoracic trauma after Ly$6 \mathrm{G}-\mathrm{Ab}$ treatment. Arrows indicate some of the positively stained cells, \# cortex, ${ }^{*}$ fracture gap, scale bar $=100 \mu \mathrm{m}$.

and MCP-1 at $6 \mathrm{~h}$ (Fig. 7). The number of CD8 ${ }^{+}$cells was unaffected (Fig. 6). In mice with combined trauma, neutrophil reduction did not significantly affect the numbers of $\mathrm{F} 4 / 80^{+}$and $\mathrm{CD}^{+}$cells and cytokine levels (Fig. 5-7).

\section{Fracture-healing outcome}

Confirming our previous studies (Kemmler et al., 2015; Recknagel et al., 2011), the additional thoracic trauma significantly impaired fracture healing as demonstrated by a significantly reduced bending stiffness and a significantly reduced relative amount of bone in the fracture callus (Fig. 8). Ly-6G-Ab treatment significantly reduced the bending stiffness and bone fraction in the callus of the mice with isolated fracture, indicating disturbed fracture healing. In the combined-trauma group, neutrophil reduction induced no significant effects. 

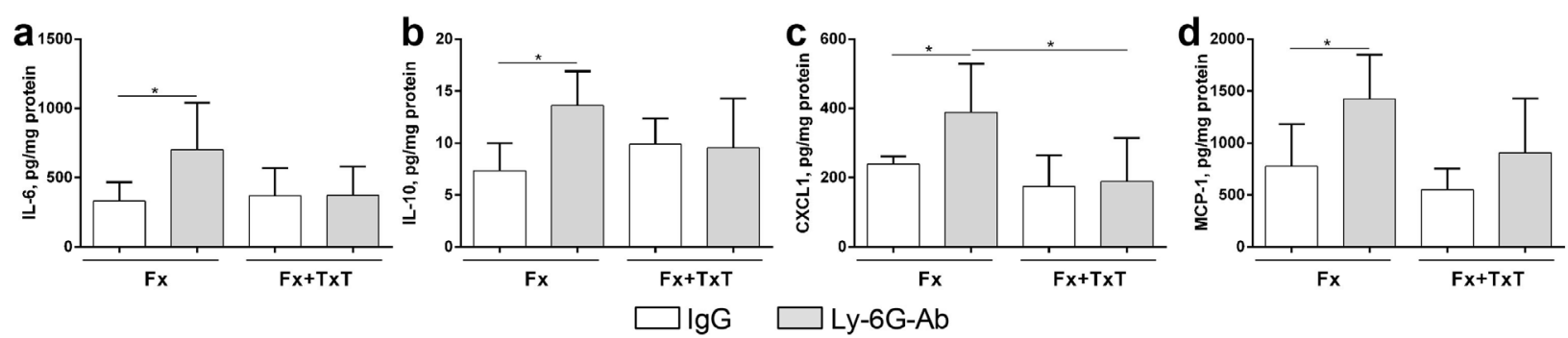

Fig. 7. Cytokine and chemokine concentrations in the fracture haematoma of control (IgG) and Ly-6G-Ab-treated mice $6 \mathrm{~h}$ after trauma. Fx: mice with isolated fracture, Fx + TxT: mice with combined fracture and thoracic trauma; $n=5-6, * p<0.05$.
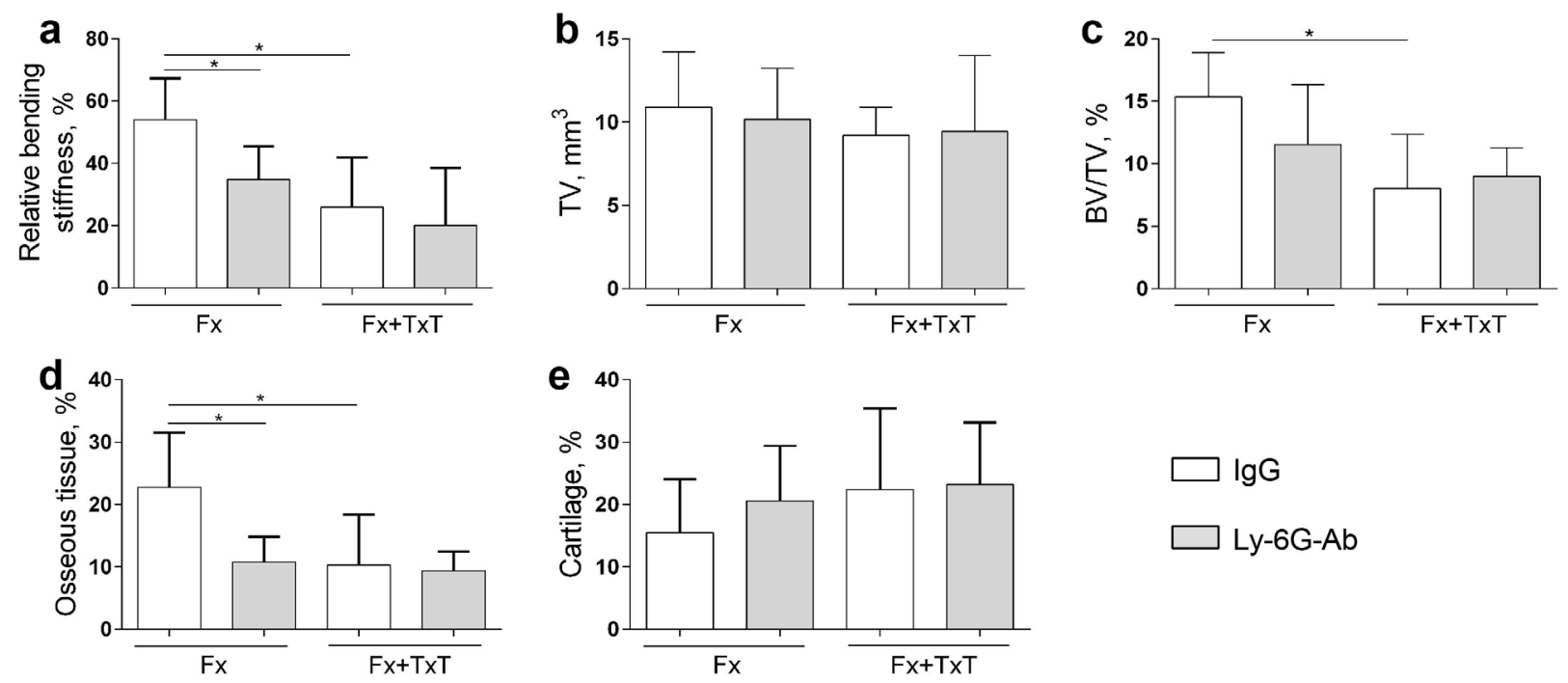

Fig. 8. Analysis of fracture healing of control (IgG) and Ly-6G-Ab-treated mice $21 \mathrm{~d}$ after trauma. Fx: mice with isolated fracture, Fx + TxT: mice with combined fracture and thoracic trauma. (a) Relative bending stiffness of fractured femora; $n=6-7$. (b) Callus tissue volume (TV) as determined by $\mu \mathrm{CT} ; n=6-7$. (c) Relative bone content (bone volume/total volume: BV/TV) in the fracture gap as determined by $\mu \mathrm{CT} ; n=6-7$. Relative amount of (d) bone and (e) cartilage in the fracture gap as determined by histomorphometric analysis using Safranin O staining; $n=5-8$, ${ }^{*} p<0.05$.

\section{Discussion}

The present study addressed the role of neutrophil granulocytes, the most abundant immune cell population in the early fracture haematoma, in 1) a mouse model of an isolated fracture inducing uneventful healing, and 2) during a compromised bone healing induced by a concomitant thoracic trauma. After isolated fracture, neutrophil reduction significantly increased the concentration of inflammatory mediators and the number of monocytes/ macrophages in the fracture haematoma and considerably impaired bone regeneration. These results suggest that undisturbed neutrophil function is crucial to initiate downstream responses leading to fracture repair. In the model of compromised fracture healing, neutrophil reduction did not provoke significant effects, indicating that these cells are presumably not a primary inducer of disturbed bone regeneration after severe trauma.

First, we aimed to analyse the role of neutrophils in uneventful fracture healing. We used young mice with excellent regenerative capacity (Lu et al., 2005) and a well-established isolated fracture model, which allows uncomplicated healing (Kemmler et al., 2015; Röntgen et al., 2010). To reduce neutrophil numbers, mice were treated with a specific anti-mouse Ly-6G antibody (Daley et al., 2008). In agreement with others (Daley et al., 2008), we found a significant reduction of neutrophil counts in the circulation on days 1-8 after Ly6G-Ab injection, a timeframe that covers the entire inflammatory period after fracture in mice (Kemmler et al., 2015). Neutrophil (Ly- $6 \mathrm{G}^{+}$cell) numbers were also significantly reduced in the fracture haematoma even in the presence of locally enhanced chemoattractants, confirming a significant reduction of neutrophil numbers.

Notably, the reduction of neutrophils in the fracture haematoma was associated with significantly increased local concentrations of IL-6 and IL-10, key cytokines in the immune response after fracture (Gerstenfeld et al., 2003), CXCL1, a neutrophil chemoattractant (Sadik et al., 2011), and MCP-1 (CCL2), a chemotactic factor for monocytes (Deshmane et al., 2009). Associated with these findings was an enhanced and prolonged infiltration by F4/80 $0^{+}$monocytes/macrophages, which may be the main source of the increased inflammatory mediators (Deshmane 
et al., 2009; Yang et al., 2014). In good agreement with this, we found increased concentrations of the monocyte chemoattractant MCP-1, which can be released by tissueresident macrophages and endothelial cells as well as by osteoblasts and osteoclasts in damaged bone (Kim et al., 2005; Li et al., 2007). The enhanced invasion of $\mathrm{F} 4 / 80^{+}$ cells to the fracture site in Ly-6G-Ab-treated mice was unexpected, because neutrophils are thought to promote monocyte invasion in injured tissues by the secretion of soluble factors, including azurocidin and cathepsin G, and "find-me" signals, for example, 19 ribosomal protein dimer and lysophosphatidylserine released by apoptotic neutrophils (Kolaczkowska and Kubes, 2013; Soehnlein et al., 2009). F4/80 cells may well be recruited by excessive alarmin concentrations, which would otherwise be cleared by neutrophils. It is known that monocytes/macrophages are attracted by PAMPs and DAMPs (Yang et al., 2014). The observed shift to an inflammatory monocyte/macrophagedominated immune response resulted in poor bone healing as demonstrated by significantly reduced bone content and mechanical properties of the fracture callus after $21 \mathrm{~d}$. These results imply that neutrophils may contribute to the resolution of inflammation in the early fracture callus. Supporting this suggestion, aggregated NETs were reported to degrade inflammatory cytokines, thus ameliorating or terminating inflammatory cascades (Kobayashi, 2015). Neutrophils also produce anti-inflammatory lipid mediators, including lipoxins and protectins (Wang and Arase, 2014). Furthermore, apoptotic neutrophils present "eat me" signals, including phosphatidylserine, which, once engulfed by macrophages, re-programme them from pro-inflammatory M1 to regulatory M2 phenotype, thus initiating repair (Headland and Norling, 2015).

Other authors found no or only marginal improvement of bone healing after neutrophil depletion (Chung et al., 2006; Grøgaard et al., 1990). However, a crucial drawback of these studies was the use of unspecific antiserum, which bears a high risk of influencing other immune cell populations. In agreement with the present study, Chan et al. described impaired bone healing after specifically depleting neutrophils using Ly-6G antibody. However, in contrast to our data, authors found less F4/80 cells at the fracture site (Chan et al., 2015). This discrepancy may be due to methodological differences. For example, in contrast to our study Chan et al. used female mice, which were previously shown to recruit fewer monocytes and neutrophils in a peritonitis model compared to male mice (Kay et al., 2015). Moreover, Chan et al. used a tibia fracture model, which may induce a different trauma load, and a much more flexible fracture fixation technique, which as well influences the inflammatory response in the fracture haematoma (Schmidt-Bleek et al., 2012). Nevertheless, our results and the study of Chan et al. (Chan et al., 2015) suggest that neutrophils are crucial to induce downstream events, which finally lead to efficient bone repair.

In the present study, we also investigated whether neutrophils play a pathomechanistic role in compromised bone healing, which is often observed in multiply injured patients, who develop a systemic inflammatory response due to severe tissue injury (Karladani et al., 2001).
Neutrophils are strongly activated in posttraumatic systemic inflammation, clearing PAMPs and DAMPs, and facilitating the recruitment of monocytes. However, they can also damage bystander tissues via the unspecific release of reactive oxygen species and matrix metalloproteinases and excessive NETosis (Hazeldine et al., 2014; Kolaczkowska and Kubes, 2013). To induce compromised bone healing, we combined the fracture with an additional thoracic trauma (Kemmler et al., 2015; Recknagel et al., 2011). The concomitant thoracic trauma induced pulmonary inflammation, indicated by a significant increase of inflammatory mediators in the BAL fluids as well as neutrophil and macrophage recruitment to the lung. However, we did not observe any significant increase in the blood levels of inflammatory mediators. Most probably, the chosen $6 \mathrm{~h}$ observation time-point was beyond the inflammatory peak response. In our previous study, systemic inflammation was clearly detectable at $3 \mathrm{~h}$ after injury (Kemmler et al., 2015). Nevertheless, in agreement with our previous data (Kemmler et al., 2015; Recknagel et al., 2011), fracture healing was significantly disturbed in the combined trauma group as demonstrated by significantly decreased bone formation and reduced biomechanical properties of the fracture callus. Because we had previously found increased recruitment of neutrophils to the fracture haematoma (Kemmler et al., 2015; Recknagel et al., 2013), we hypothesised that neutrophils may play a pathomechanistic role. In the present study, the neutrophil number in the fracture haematoma was also increased at day 1 , while $\mathrm{F} 4 / 80^{+}$cells were increased at day 3 , confirming that inflammation at the fracture site was affected by the additional thoracic trauma. However, the reduction of neutrophils did not provoke any effect on the inflammatory response at the fracture site nor on the fracture healing outcome in the combined-trauma group, indicating that neutrophils presumably are not the primary inducers of disturbed bone regeneration after severe trauma. Nevertheless, neutrophil reduction ameliorated pulmonary inflammation as demonstrated by a decreased concentration of inflammatory cytokines in the BAL fluids and a reduced amount of F4/80 protein in tissue homogenates. Confirming these data, others authors also reported mitigated pulmonary damage after depletion of neutrophils in thoracic trauma-induced acute lung injury (Perl et al., 2012a; Perl et al., 2012b). The discrepancy of the effects of neutrophil reduction in the fracture haematoma and the lung could be explained by the compartmentalisation of the immune response, because similar inflammatory stimuli can lead to completely different immune response patterns in different tissues (Cavaillon and Annane, 2006; Chinnaiyan et al., 2001). Neutrophils can be activated to a different extend depending on the severity of trauma and the microenvironment in the affected organ. For example, in lungs the neutrophil reduction protected mice against alveolar-capillary dysfunction (Perl et al., 2012a; Perl et al., 2012b), whereas it simultaneously ameliorated immunosuppressive response of splenocytes and Kupffer cells (Perl et al., 2012a; Perl et al., 2012b). Further research is needed to decipher the underlying mechanisms for the diverse neutrophil function in different organs after trauma. 


\section{Conclusions}

The present study demonstrated that undisturbed neutrophil recruitment and function in the inflammatory phase of fracture healing is crucial to initiate downstream responses leading to fracture repair, because bone regeneration was considerably disturbed after neutrophil reduction. However, neutrophils appear to play a minor pathomechanistic role in compromised fracture healing induced by an additional thoracic trauma. Further studies are needed to unravel the discrepant results on the role of neutrophils in the local inflammatory responses in the lungs and bone.

\section{Acknowledgements}

The study was funded by the German Research Foundation in the context of the Clinical Research Unit (KFO200, IG-18/14-2) and of the Collaborative Research Centre (CRC1149).

We would like to thank Sevil Essig, Stephanie Riesemann, Uschi Maile, Marion Tomo, Helga Bach, and Iris Baum for their excellent technical assistance. We are also deeply grateful to Sonja Braumüller for her help with the cytokine analysis.

\section{References}

Bastian O, Pillay J, Alblas J, Leenen L, Koenderman L, Blokhuis T (2011) Systemic inflammation and fracture healing. J Leukoc Biol 89: 669-673.

Bhandari M, Tornetta P, 3rd, Sprague S, Najibi S, Petrisor B, Griffith L, Guyatt GH (2003) Predictors of reoperation following operative management of fractures of the tibial shaft. J Orthop Trauma 17: 353-361.

Bozlar M, Aslan B, Kalaci A, Baktiroglu L, Yanat AN, Tasci A (2005) Effects of human granulocyte-colony stimulating factor on fracture healing in rats. Saudi Med J 26: 1250-1254.

Butterfield TA, Best TM, Merrick MA (2006) The dual roles of neutrophils and macrophages in inflammation: $A$ critical balance between tissue damage and repair. J Athl Train 41: 457-465.

Cavaillon JM, Annane D (2006) Compartmentalization of the inflammatory response in sepsis and SIRS. J Endotoxin Res 12: 151-170.

Chan JK, Glass GE, Ersek A, Freidin A, Williams GA, Gowers K, Espirito Santo A, Jeffery R, Otto WR, Poulsom R, Feldmann M, Rankin SM, Horwood NJ, Nanchahal J (2015) Low-dose TNF augments fracture healing in normal and osteoporotic bone by up-regulating the innate immune response. EMBO Mol Med 7: 547-561.

Chinnaiyan AM, Huber-Lang M, Kumar-Sinha C, Barrette TR, Shankar-Sinha S, Sarma VJ, Padgaonkar VA, Ward PA(2001) Molecular signatures of sepsis: multiorgan gene expression profiles of systemic inflammation. Am J Pathol 159: 1199-1209.

Chung R, Cool JC, Scherer MA, Foster BK, Xian CJ (2006) Roles of neutrophil-mediated inflammatory response in the bony repair of injured growth plate cartilage in young rats. J Leukoc Biol 80: 1272-1280.

Claes L, Recknagel S, Ignatius A (2012) Fracture healing under healthy and inflammatory conditions. Nat Rev Rheumatol 8: 133-143.

Daley JM, Thomay AA, Connoly MD, Reichner JS, Albine JE (2008) Use og Ly6G-specific monoclonal antibody to deplete neutrophils in mice. J Leukoc Biol 83: 64-70.

Deshmane SL, Kremlev S, Amini S, Sawaya BE (2009) Monocyte chemoattractant protein-1 (MCP-1): An Overview. J Interferon Cytokine Res 29: 313-326.

Furze RC, Rankin SM (2008) Neutrophil mobilization and clearance in the bone marrow. Immunology 125: 281288.

Gerstenfeld LC, Cullinane DM, Barnes GL, Graves DT, Einhorn TA (2003) Fracture healing as a post-natal developmental process: molecular, spatial, and temporal aspects of its regulation. J Cell Biochem 88: 873-884.

Giannoudis PV, Einhorn TA, Marsh D (2007) Fracture healing: the diamond concept. Injury 38 Suppl 4: S3-S6.

Grøgaard B, Gerdin B, Reikerås O (1990) The polymorphonuclear leukocyte: has it a role in fracture healing? Arch Orthop Trauma Surg 5: 268-271.

Hak DJ, Fitzpatrick D, Bishop JA, Marsh JL, Tilp S, Schnettler R, Simpson H, Alt V (2014) Delayed union and nonunions: epidemiology, clinical issues, and financial aspects. Injury 45: S3-S7.

Hazeldine J, Hampson P, Lord JM (2014) The impact of trauma on neutrophil function. Injury 45: 1824-1833.

Headland SE, Norling LV (2015) The resolution of inflammation: Principles and challenges. Semin Immunol 27: 149-160.

Karladani AH, Granhed H, Kärrholm J, Styf J (2001) The influence of fracture etiology and type on fracture healing: a review of 104 consecutive tibial shaft fractures. Arch Orthop Trauma Surg 121: 325-328.

Kay E, Gomez-Garcia L, Woodfin A, Scotland RS, Whiteford JR (2015) Sexual dimorphisms in leukocyte trafficking in a mouse peritonitis model. J Leukoc Biol 98: $805-817$.

Kemmler J, Bindl R, McCook O, Wagner F, Gröger M, Wagner K, Scheuerle A, Radermacher P, Ignatius A (2015) Exposure to $100 \%$ oxygen abolishes the impairment of fracture healing after thoracic trauma. PLoS One. doi: 10.1371/journal.pone.0131194.

Kim MS, Day CJ, Morrison NA (2005) MCP-1 is induced by receptor activator of nuclear factor- $\{$ kappa $\}$ $\mathrm{B}$ ligand, promotes human osteoclast fusion, and rescues granulocyte macrophage colony-stimulating factor suppression of osteoclast formation. J Biol Chem 280: 16163-16169.

Knöferl MW, Liener UC, Seitz DH, Perl M, Brückner UB, Kinzl L, Gebhard F (2003) Cardiopulmonary, histological, and inflammatory alterations after lung contusion in a novel mouse model of blunt chest trauma. Shock 19: 519-525.

Kobayashi Y (2015) Neutrophil biology: an update. EXCLI J 14: 220-227. 
Kolaczkowska E, Kubes P (2013) Neutrophil recruitment and function in health and inflammation. Nat Rev Immunol 13: 159-175.

Kolar P, Gaber T, Perka C, Duda GN, Buttgereit F (2011) Human early fracture hematoma is characterized by inflammation and hypoxia. Clin Orthop Relat Res 469: 3118-3126.

Kolar P, Schmidt-Bleek K, Schell H, Gaber T, Toben D, Schmidmaier G, Perka C, Buttgereit F, Duda GN (2010) The early fracture hematoma and its potential role in fracture healing. Tissue Eng Part B Rev 16: 427-434.

Lefering R, Paffrath T, Neunaber U (2013) Trauma register DGU annual report. German trauma society (DGU), committee on emergency medicine, intensive care and trauma management (Section NIS), and AUC Academy of Trauma Surgery. $\S$

Li X, Qin L, Bergenstock M, Bevelock LM, Novack DV, Partridge NC (2007) Parathyroid hormone stimulates osteoblastic expression of MCP-1 to recruit and increase the fusion of pre/osteoclasts. J Biol Chem 282: 3309833106.

Lu C, Miclau T, Hu D, Hansen E, Tsui K, Puttlitz C, Marcucio RS (2005) Cellular basis for age-related changes in fracture repair. J Orthop Res 23: 1300-1307.

Morgan EF, Mason ZD, Chien KB, Pfeiffer AJ, Barnes GL, Einhorn TA, Gerstenfeld LC (2009) Micro-computed tomography assessment of fracture healing: Relationships among callus structure, composition, and mechanical function. Bone 44: 335-344.

Pape HC, Marcucio R, Humphrey C, Colnot C, Knobe M, Harvey EJ (2010) Trauma-induced inflammation and fracture healing. J Orthop Trauma 24: 522-525.

Parfitt AM, Drezner MK, Glorieux FH, Kanis JA, Malluche H, Meunier PJ, Ott SM, Recker RR (1987) Bone histomorphometry: standardization of nomenclature, symbols and units. Report of the ASBMR histomorphometry nomenclature committee. J Bone Miner Res 2: 595-610.

Perl M, Hohmann C, Denk S, Kellermann P, Lu D, Braumüller S, Bachem MG, Thomas J, Knöferl MW, Ayala A, Gebhard F, Huber-Lang MS (2012a) Role of activated neutrophils in chest trauma-induced septic acute lung injury. Shock 38: 98-106.

Perl M, Kieninger M, Huber-Lang M, Gross HJ, Bachem MG, Braumüller S, Gebhard F, Ayala A, Knöferl MW (2012b) Divergent effects of activated neutrophils on inflammation, Kupffer cell/splenocyte activation, and lung injury following blunt chest trauma. Shock 37: 210-218.

Recknagel S, Bindl R, Brochhausen C, Göckelmann M, Wehner T, Schoengraf P, Huber-Lang M, Claes L, Ignatius A (2013) Systemic inflammation induced by a thoracic trauma alters the cellular composition of the early fracture callus. J Trauma Acute Care Surg 74: 531-537.

Recknagel S, Bindl R, Kurz J, Wehner T, Ehrnthaller C, Knöferl MW, Gebhard F, Huber-Lang M, Claes L, Ignatius A (2011) Experimental blunt chest trauma impairs fracture healing in rats. J Orthop Res 29: 734-739.

Röntgen V, Blakytny R, Matthys R, Landauer M, Wehner T, Göckelmann M, Jermendy P, Amling M, Schinke T, Claes L, Ignatius A (2010) Fracture healing in mice under controlled rigid and flexible conditions using an adjustable external fixator. J Orthop Res 28: 1456-1462.

Sadik CD, Kim ND, Luster AD (2011) Neutrophils cascading their way to inflammation. Trends Immunol 32: 452-460.

Schmidt-Bleek K, Kwee BJ, Mooney DJ, Duda GN (2015) Boon and bane of inflammation in bone tissue regeneration and its link with angiogenesis. Tissue Eng Part B Rev 21: 354-364.

Schmidt-Bleek K, Schell H, Schulz N, Hoff P, Perka C, Buttgereit F, Volk HD, Lienau J, Duda GN (2012) Inflammatory phase of bone healing initiates the regenerative healing cascade. Cell Tissue Res 347: 567573.

Soehnlein O, Lindbom L, Weber C (2009) Mechanisms underlying neutrophil-mediated monocyte recruitment. Blood 114: 4613-4623.

Tian M, Qing C, Niu Y, Dong J, Cao X, Song F, Ji X, Lu S (2014) The relationship between inflammation and impaired wound healing in a diabetic rat burn model. J Burn Care Res: 37: e115-124.

Wang J, Arase H (2014) Regulation of immune responses by neutrophils. Ann N Y Acad Sci 1319: 66-81.

Wong SL, Demers M, Martinod K, Gallant M, Wang Y, Goldfine AB, Kahn CR, Wagner DD (2015) Diabetes primes neutrophils to undergo NETosis, which impairs wound healing. Nat Med 21: 815-819.

Yang J, Zhang L, Yu C, Yang XF, Wang H (2014) Monocyte and macrophage differentiation: circulation inflammatory monocyte as biomarker for inflammatory diseases. Biomark Res 2: 1-9.

$\S$ This report is available on-line from the following URL: http://www.dgu-online.de/fileadmin/published_content/5. Qualitaet_und_Sicherheit/PDF/2013_TR_DGU_annual_ report.pdf

\section{Discussion with Reviewers}

Reviewer I: The increase in macrophages upon neutrophil depletion in uneventful healing could be understood as a rescue mechanism, please elaborate?

Would you explain the heightened pro-inflammatory reaction with the higher macrophage count and their persistent M1 phenotype?

Authors: Neutrophils are known to act as the first line of defence by clearing PAMPs and DAMPs after tissue trauma. After neutrophil reduction we found an enhanced and prolonged infiltration of the fracture hematoma by $\mathrm{F} 4 / 80^{+}$cells. The $\mathrm{F} 4 / 80^{+}$cells might be recruited by excessive alarmin concentrations, which would otherwise be cleared by neutrophils. Associated with these findings was a significant increase of inflammatory mediators suggesting that the $\mathrm{F} 4 / 80^{+}$cells were indeed M1 macrophages. The persistence of these cells indicates delayed resolution of inflammation, which might be responsible for the observed disturbed fracture healing. To finally prove the macrophage phenotype simultaneous co-staining of several M1 markers such as iNOS and CD86 
is required. Thus, further experiments using for example flow cytometry would be necessary. These analyses require an additional number of animals and are beyond the scope of the present paper.

Reviewer II: PMN are bad in polytrauma as they damage lung tissue but may be good for fracture healing, as you studied. Please discuss this dichotomy.

Authors: Neutrophils can be activated to a different extend depending on the severity of trauma and the microenvironment in the affected organ. For example, in lungs PMN were suggested to contribute to the development of the acute lung injury because their reduction protected against alveolar-capillary dysfunction (Perl et al., 2012a). In contrast, it simultaneously ameliorated immunosuppressive response of splenocytes and Kupffer cells (Perl et al., 2012b). Further research is needed to decipher the underlying mechanisms for the diverse neutrophil function in different organs after trauma.

Reviewer II: Which factors of the PMN may be important for the fracture healing?

Authors: We suggest that a balanced neutrophil response contributes to the resolution of inflammation in the early fracture callus by clearing PAMPs and DAMPs, by the degradation of inflammatory cytokines, and by the production of anti-inflammatory lipid mediators. Furthermore, apoptotic neutrophils present "eat me" signals, which, once engulfed by macrophages, reprogramme them from pro-inflammatory M1 to regulatory M2 macrophages (Kobayashi, 2015; Wang and Arase, 2014; Headland and Norling, 2015).

Reviewer II: Why PMN reduction didn't have a difference when thorax trauma was present? You mentioned only a reduction of $59 \%$ of PMN. How was the absolute number of PMN? This may be more when thorax trauma is present. Authors: We did not measure the absolute neutrophil numbers in the blood. However, in the fracture haematoma neutrophil numbers were similar in both the isolated fracture and combined trauma group after Ly-6G-Ab treatment (Fig. 4). Bone healing was similar in these two groups as well. Therefore, we suggested that local neutrophil recruitment might not play a crucial pathomechanistic role in compromised fracture healing induced by an additional thoracic trauma.

Editor's note: The Scientific Editor responsible for this paper was Martin Stoddart. 